\title{
Echocardiographic findings among children with pectus excavatum
}

\author{
Esra Akyüz Özkan ${ }^{1}$, Hashem E. Khosroshahi², Mahmut Kılıç³, Bayram Metin ${ }^{4}$, Halil İ. Serin ${ }^{5}$
}

\footnotetext{
1) Department of Pediatrics, Bozok University Medical Faculty, Yozgat, Turkey

${ }^{2)}$ Department of Pediatric Cardiology, Bozok University Medical Faculty, Yozgat, Turkey

3) Department of Public Health, Bozok University Medical Faculty, Yozgat, Turkey

4) Department of Thoracic Surgery, Bozok University Medical Faculty, Yozgat, Turkey

${ }^{5)}$ Department of Radiology, Bozok University Medical Faculty, Yozgat, Turkey
}

\begin{abstract}
Objective: Pectus excavatum (PE) is characterized by the posterior displacement of inferior sternum and adjacent cartilages and is the most common congenital chest wall deformity. We aimed to investigate right and left ventricular functions and its correlation with pectus severity index in children.

Methods: Echocardiography was performed in 32 children with PE and 40 healthy controls. The following parameters were monitored: Left and right ventricular (LV, RV) ejection fraction (EF), ejection time (ET), stroke volume (SV), shortening fraction (SF), mitral and tricuspid early (E) and late (A) ventricular filling velocities and deceleration time (DT), median pulmonary arterial pressure (PAPm), aorta and pulmonary acceleration time (Ao-AT, PA-AT), RV work index (RVWI), isovolumetric myocardial acceleration (IVMA), and LV pulmonary ejection period (PEPLV). To assess the severity of pectus, Haller Index (HI) was calculated by thorax computed tomography.

Results: There was no significant difference regarding averages of the body surface area (BSA) between the groups. The arithmetic averages of the EF-Left, ET-Left, ET-Right, SV-Left, TV-DT, PA-AT, and PEPLV were higher in children with PE than in controls, but SF-Left, SF-Right, MV-A, and IVMA were found to be lower. Haller Index (HI) value in children with PE was $2.00-4.93(2.62 \pm 0.56)$. We failed to demonstrate any statistically significant relationship between the cardiac data of children with PE and HI.
\end{abstract}

Conclusion: Despite the fact that most children had only a mild or moderate form of PE, the RV and LV functions were affected.

Keywords: Children, echocardiography, left ventricular function, pectus excavatum, right ventricular function 


\section{Introduction}

Pectus excavatum (PE), or funnel chest, is one of the most common congenital skeletal deformities. Characterized by an inward depression of the sternum, it is seen in approximately 1 in every 400 male births. ${ }^{[1]}$ Pectus anomalies can be diagnosed at an early age and are three times more common in boys. ${ }^{[2]}$

The etiology of PE is unknown; it is often seen as isolated anomalies. The primary deformity is dystrophic costal cartilage growth along with the sternum depression. Pectus deformity manifests as subtle abnormalities associated with collagen morphology of children's costal cartilages, but the causal significance of this is not precisely known. Approximately one-third of cases have a positive family history of chest wall deformity. ${ }^{[3]}$

In addition to cosmetic problems, posterior angulation of the sternum and rib cartilages may cause changes in the rotation and location of the heart and may lead to cardiorespiratory function abnormalities. ${ }^{[4]}$ Some studies demonstrated a significant compromise of cardiac or pulmonary function $\mathrm{S}^{[5,6]}$, whereas other studies showed no change in cardiac functions. ${ }^{[7]}$ Decreased cardiac output, mitral valve prolapse (MVP), and dysrhythmias are considered the primary cardiac effects of PE. Compression of the heart, in particular of the right atrium and ventricle by the chest wall, results in incomplete filling and decreased stroke volume, and eventually in decreased cardiac output. ${ }^{[5,6]}$ Similarly, compression of the right ventricle (RV) by the chest wall can lead to patient symptoms including dyspnea and chest pain with exertion. ${ }^{[8,9]}$ The chest deformity may also cause compression on the vena cava inferior. ${ }^{[10]}$

There are conflicting studies about the effects of PE on cardiopulmonary functions. Malek et al. ${ }^{[1]}$ demonstrated that the oxygen pulse and maximum oxygen uptake were low in PE patients. Yalamanchili et al. ${ }^{[12]}$ showed in a case with PE that SV-Rt (RV stroke volume) was reduced. Haller et al. ${ }^{[13]}$ reported that cardiopulmonary functions improved after surgery. Conversely, it is reported that since these patients do not participate in social events and sports, the cardiopulmonary symptoms are related to psychological disorders. ${ }^{[14,15]}$
We aimed to investigate right and left ventricular (LV) functions more comprehensively and determine their correlation with pectus severity index in children with PE.

\section{Materials and methods}

We studied 32 pediatric patients selected randomly from those with PE but without any other congenital anomaly or disease and 40 healthy subjects. Individuals with pulmonary, renal diseases and a history of diabetes, hypertension, obesity or other systemic diseases were excluded. A full history was taken and a complete physical examination was performed by the same physician. The body height and weight of all children were recorded. Body surface area (BSA) was calculated by Mosteller formula. ${ }^{[16]}$

At the time of the other tests, an electrocardiogram was recorded for all patients. Transthoracic echocardiography was performed by a single experienced pediatric cardiologist and the following parameters were monitored:

Ejection fraction (EF) was calculated using the standard dimension cubed formula:

$\boldsymbol{E F}=(\boldsymbol{L V D D 3}-\boldsymbol{L V D S 3}) / \boldsymbol{L V D D 3}$, where LVDD and LVDS stands for LV dimension in diastole and systole respectively.

SV was calculated as: $S V=(\boldsymbol{L V O T} / 2) 2 \times V T I A o$ $\times$ 3.141. The LV outflow tract (LVOT) diameter was measured at the base of the aortic leaflet at the parasternal long axis view in echocardiography. Time velocity integral for aortic valve (VTIAo) was obtained with continual wave Doppler immediately below the aortic valve in the apical long axis view.

Aortic Doppler was used to calculate the time intervals - the pre-ejection period (PEP), i.e. the time interval from $\mathrm{Q}$ wave of ECG to the onset point of aortic Doppler flow, and the Q-T offset interval, i.e. the time interval from $\mathrm{Q}$ wave of ECG to the offset point of aortic Doppler flow.

Mitral and tricuspid early (E) and late (A) filling velocities were recorded from the apical four-chamber view with the pulse-wave Doppler during diastole. E, 
A, and deceleration time (DT) were used as both ventricular diastolic function parameters. Ejection time (ET) was calculated from the beginning to the end of the pulmonary and aortic flow.

Isovolumetric myocardial acceleration (IVMA) was calculated by dividing isovolumetric volume (IVV) by the time interval from onset of IVV to the time at peak velocity of this wave.

Mean pulmonary arterial pressure $(\mathrm{PAPm})=0.65$ $\times(P A P S+0.55)$;

$R V$ work index $(R V W I)=0.136 \times(P A P m-R A P)$ $\times S V$;

Shortening fraction $(S F)=L V$ end-diastolic $-L V$ end-systolic / LV end-diastolic diameter;

Haller Index $(\mathrm{HI})=$ A / B where A stands for transverse diameter at the deepest level of deformity, and $\mathrm{B}$ for anterior-posterior diameter of the same level. An HI $<2.5$ was considered as mild, $\mathrm{HI}=2.5-3.2$ as moderate, and $\mathrm{HI}>3.2$ as severe deformity. The $\mathrm{A}$ and $\mathrm{B}$ diameters of our PE subjects were calculated by thorax computed tomography.

\section{Statistical analyses}

The statistical analyses were performed using Statistical Package for the Social Sciences (SPSS). Data were analyzed by independent $t$ test and Analysis of covariance (ANCOVA) test. The arithmetic means of cardiac measurements of children with pectus deformity and of the control group were compared according to independent $t$ test. Important parameters between groups as determined by independent $t$ test were subjected to ANCOVA multiple analyses. Cardiac parameters can be affected by age and the BSA, so these two factors are taken as covariate variables to multi ANCOVA analysis. In ANCOVA analysis, pectus and control group were taken as fixed factors; age and BSA were taken as covariate variables. $\mathrm{HI}$ arithmetic average and correlation values of $\mathrm{HI}$ and the cardiac parameters were investigated.

\section{Results}

32 children (23 male, 9 female) with PE deformity and 40 (17 male, 23 female) healthy controls were included in the study. There was no significant difference regarding average of the age and body surface area (BSA) between the groups.

The arithmetic averages of the EF-Lft, ET-Lft, ETRt, SV-Lft, TV-DT, PA-AT and PEPLV were found to be higher in children with PE than in controls (Table 1).

ANCOVA analysis revealed that, when age and BSA were taken as covariate variables and pectus and control group as fixed factors, all parameters mentioned above were found to be statistically significant (Table 2).

The arithmetic average of the SF-Lft, SF-Rt, MV-A and IVMA were found to be lower in children with PE than in controls (Table 1). According to ANCOVA analysis, when age and BSA were taken as covariate variables and pectus and control group as fixed factors, all parameters except for MV-A were found to be statistically significant. These differences in SF-Lft of $48.5 \%$, in SF-Rt of $19.4 \%$, in IVMA of $8.2 \%$, and in EF-Rt of $7.2 \%$ can be attributed to pectus disease (Table 2).

HI value among children with PE ranged between 2.00 and 4.93 (2.62 \pm 0.56$)$. Of the total of 32 patients with PE, 14 showed mild, 15 moderate, and 3 severe deformities. We found no statistically significant correlation between $\mathrm{HI}$ and cardiac parameters among children with PE.

\section{Discussion}

Although PE can be viewed as a slight problem, it can lead to much more than a cosmetic deformity. Volume reduction and cardiac chest compression can lead to a reduction in cardiopulmonary function and physical capacity. Symptoms rarely appear in early childhood but increase with age. ${ }^{[17]}$ The chest wall elasticity decreases, stiffness increases, heart deviation to the left decreases, and pressure on the heart increases, with corresponding increase of symptoms with age in PE patients.

RV dysfunction can be seen in patients with PE. Ventricles share a common septum and are within the same pericardial cavity. This relationship between the ventricles also causes similar changes in both systolic and diastolic functions. ${ }^{[18]}$ The compression on the RV 
also can cause changes in the size and function of the LV. Cardiac output and SV were demonstrated to be reduced in pectus patients and improved after corrective surgery. ${ }^{[19]}$ Gürsu et al. ${ }^{[20]}$ found lower EF-Lft in the PE group and also revealed that there was an inverse relationship between EF-Lft and SF by HI. EF-Lft and SF-Lft were significantly reduced by increasing HI, but LV end-diastolic volume showed no significant change.
Another study ${ }^{[21]}$ demonstrated, using transesophageal echocardiography, that RV end-diastolic size and EFLft were increased after the surgery. Bawazir et al. ${ }^{[22]}$ showed that after the pectus corrective surgery, the LV cardiac output and index improved and was maintained thereafter. In their meta-analysis, Malek et al. ${ }^{[23]}$ indicated that the LV function was increased after surgery. Lyons et al. ${ }^{[24]}$ reported the pattern of the RV pressure in

Table 1-1. Echocardiographic findings of children with pectus excavatum compared to the control group

\begin{tabular}{|c|c|c|c|c|c|c|}
\hline Cardiac parameters & Groups & $\mathbf{N}$ & Mean & Std. Deviation & $\mathbf{t}$ & p \\
\hline \multirow{2}{*}{ Age } & Pectus & 32 & 11.16 & 3.15 & -1.215 & .228 \\
\hline & Control & 40 & 12.08 & 3.21 & & \\
\hline \multirow{2}{*}{ BSA } & Pectus & 32 & 1.248 & .301 & -1.435 & .156 \\
\hline & Control & 40 & 1.359 & .347 & & \\
\hline \multirow{2}{*}{ ET-Lft (msec) } & Pectus & 32 & 292.968 & 21.159 & 7.275 & $<.001$ \\
\hline & Control & 40 & 245.800 & 30.898 & & \\
\hline \multirow{2}{*}{ ET-Rt (msec) } & Pectus & 32 & 317.161 & 26.303 & 11.171 & $<.001$ \\
\hline & Control & 40 & 241.100 & 30.006 & & \\
\hline \multirow{2}{*}{ SV-Lft (ml) } & Pectus & 32 & 61.398 & 20.000 & 2.541 & 114 \\
\hline & Control & 40 & 65.989 & 23.084 & & \\
\hline \multirow{2}{*}{ SV-Rt (ml) } & Pectus & 32 & 66.545 & 22.769 & .334 & .739 \\
\hline & Control & 40 & 64.700 & 23.655 & & \\
\hline \multirow{2}{*}{ SF-Lft (\%) } & Pectus & 32 & .222 & .074 & -8.243 & $<.001$ \\
\hline & Control & 40 & .342 & .049 & & \\
\hline \multirow{2}{*}{ SF-Rt (\%) } & Pectus & 32 & .289 & .092 & -4.206 & $<.001$ \\
\hline & Control & 40 & .387 & .101 & & \\
\hline \multirow{2}{*}{ MV-A (cm/sec) } & Pectus & 32 & 53.000 & 9.837 & -2.000 & .049 \\
\hline & Control & 40 & 58.600 & 13.163 & & \\
\hline \multirow{2}{*}{ MV-E (cm/sec) } & Pectus & 32 & 103,56 & 11,528 & -.244 & .808 \\
\hline & Control & 40 & 104,28 & 12,926 & & \\
\hline \multirow{2}{*}{ MV-DT (msec) } & Pectus & 32 & 207,88 & 54,289 & 1.306 & .196 \\
\hline & Control & 40 & 192,50 & 45,608 & & \\
\hline
\end{tabular}


patients with PE. Peterson et al. ${ }^{[25]}$ found significant improvement in RV end-diastolic volume and EF-Rt and also increments in LV end-diastolic volume index and SV index after pectus surgery. Saleh et al. ${ }^{[26]}$ found that both EF-Lft and EF-Rt were lower and RV end-systolic volume was significantly higher in PE patients.

They did not detect any significant correlation be- tween the EF-Rt and EF-Lft and pectus index. They also did not find any differences in EF-Lft, SF-Lft, aortopulmonary circulation time, or pulmonary flow indices between PE patients and controls. They suggested that the high end-systolic volume caused reduced EF-Rt. To assess ventricular global functions, EF is the commonly used measurement. Reduced EF generally reflects decreased contractility. Because EF depends on loading

Table 1-2. Echocardiographic findings of children with pectus excavatum compared to the control group

\begin{tabular}{|c|c|c|c|c|c|c|}
\hline Cardiac parameters & Groups & $\mathbf{N}$ & Mean & Std. Deviation & $\mathbf{t}$ & $\mathbf{p}$ \\
\hline \multirow{2}{*}{ TV-A (cm/sec) } & Pectus & 32 & 47,84 & 10,913 & -1.595 & 117 \\
\hline & Control & 40 & 51,48 & 7,646 & & \\
\hline \multirow{2}{*}{ TV-E (cm/sec) } & Pectus & 32 & 82,97 & 15,233 & -1.476 & 145 \\
\hline & Control & 40 & 87,78 & 12,413 & & \\
\hline \multirow{2}{*}{ TV-DT (msec) } & Pectus & 32 & 214.938 & 59.976 & 1.993 & .050 \\
\hline & Control & 40 & 189.875 & 46.765 & & \\
\hline \multirow{2}{*}{ PAPm (mm/Hg) } & Pectus & 32 & 12,996 & 3,526 & .475 & 638 \\
\hline & Control & 40 & 12,700 & ,000 & & \\
\hline \multirow{2}{*}{ PA-AT (msec) } & Pectus & 32 & 129.513 & 19.639 & 3.252 & .002 \\
\hline & Control & 40 & 115.600 & 16.400 & & \\
\hline \multirow{2}{*}{ PA-Acc } & Pectus & 32 & 8.912 & 2.072 & -1.075 & 286 \\
\hline & Control & 40 & 9.473 & 2.301 & & \\
\hline \multirow{2}{*}{ Ao-Acc } & Pectus & 32 & 13,289 & 4,152 & -.377 & .708 \\
\hline & Control & 40 & 13,616 & 3,102 & & \\
\hline \multirow{2}{*}{ Ao-AT (msec) } & Pectus & 32 & 94,067 & 17,031 & 1.203 & .233 \\
\hline & Control & 40 & 89,425 & 15,152 & & \\
\hline \multirow{2}{*}{ RVWI (gm-m/m2) } & Pectus & 32 & 4.181 & 2.011 & 1.189 & 238 \\
\hline & Control & 40 & 4.721 & 1.839 & & \\
\hline \multirow{2}{*}{ IVMA (cm/msec2) } & Pectus & 32 & .067 & .027 & -2.374 & .020 \\
\hline & Control & 40 & .083 & .029 & & \\
\hline \multirow{2}{*}{ PEPLV (msec) } & Pectus & 32 & 71.813 & 12.238 & 3.195 & .002 \\
\hline & Control & 40 & 63.050 & 11.001 & & \\
\hline \multicolumn{7}{|c|}{$\begin{array}{l}\text { SD: Standard Deviation. BSA: Body surface area, EF: Ejection fraction, ET: Ejection time, SV: Stroke volume, SF: Shortening } \\
\text { fraction, MV: Mitral valve, TV: Tricuspid valve, DT: Deceleration time, PAPm: median pulmonary arterial pressure, } \\
\text { PA-AT: Pulmonary artery acceleration time, Ao: aorta, Acc: acceleration, RVWI: Right ventricular work index, } \\
\text { IVMA: isovolumetric myocardial acceleration, PEPLV: Left ventricular pulmonary ejection period, Rt: right, Lft: left. }\end{array}$} \\
\hline
\end{tabular}


Table 2. The cardiac measurements were analyzed by ANCOVA according to age, BSA vs. groups

\begin{tabular}{|c|c|c|c|c|c|c|}
\hline $\begin{array}{c}\text { Cardiac } \\
\text { measurements }\end{array}$ & Variables & $\mathbf{F}$ & Sig. & $\begin{array}{l}\text { Partial Eta } \\
\text { Squared }\end{array}$ & $\mathbf{R} 2$ & Adj. $R 2$ \\
\hline \multirow{3}{*}{ EF-Rt (\%) } & Age & 1.56 & .216 & & & \\
\hline & BSA & .48 & .490 & & & \\
\hline & Groups & 5.29 & .025 & .072 & .108 & .069 \\
\hline \multirow{3}{*}{ ET-Lft (msec) } & Age & 4.12 & .046 & .058 & & \\
\hline & BSA & .35 & .554 & & & \\
\hline & Groups & 63.06 & $<.001$ & .485 & .507 & .485 \\
\hline \multirow{3}{*}{ ET-Rt (msec) } & Age & .249 & .620 & & & \\
\hline & BSA & .222 & .629 & & & \\
\hline & Groups & 130.62 & $<.001$ & .661 & .662 & .647 \\
\hline \multirow{3}{*}{ SF-Lft (\%) } & Age & .16 & .694 & & & \\
\hline & BSA & .03 & .867 & & & \\
\hline & Groups & 63.47 & $<.001$ & .483 & .495 & .472 \\
\hline \multirow{3}{*}{ SF-Rt (\%) } & Age & 2.10 & .152 & & & \\
\hline & BSA & .75 & .391 & & & \\
\hline & Groups & 16.34 & $<.001$ & .194 & .233 & .199 \\
\hline \multirow{3}{*}{ MV-A (cm/sec) } & Age & 5.11 & .027 & .070 & & \\
\hline & BSA & 4.29 & .042 & .059 & & \\
\hline & Groups & 3.84 & .054 & .054 & .121 & .082 \\
\hline \multirow{3}{*}{ TV-DT (msec) } & Age & 2.022 & .160 & & & \\
\hline & BSA & .590 & .445 & & & \\
\hline & Groups & 4.527 & .037 & .062 & .093 & .053 \\
\hline \multirow{3}{*}{ PA-AT (msec) } & Age & 1.178 & .282 & & & \\
\hline & BSA & .052 & .820 & & & \\
\hline & Groups & 12.229 & $<.001$ & .154 & .173 & .136 \\
\hline \multirow{3}{*}{ PEPLV (msec) } & Age & 3.525 & .065 & & & \\
\hline & BSA & 1.319 & .255 & & & \\
\hline & Groups & 11.268 & $<.001$ & .142 & .182 & .145 \\
\hline \multirow{3}{*}{ IVMA (cm/msec2) } & Age & 1.501 & .225 & & & \\
\hline & BSA & .579 & .449 & & & \\
\hline & Groups & 6.005 & .017 & .082 & .101 & .061 \\
\hline
\end{tabular}


conditions, and changes in preload and afterload affect the contractility, EF is not considered an accurate measurement ${ }^{[27,28]}$ Similarly, regional EF is also loaddependent and does not reveal contractility. ${ }^{[29]}$ IVMA is a measurement of ventricular contractile function that is unaffected by preload and afterload changes in a physiological range. Vogel et al. ${ }^{[30]}$ showed in a pig model that IVMA was less load-dependent than EF.

They demonstrated that IVMA was unchanged with the decrease in the preload and increase in the afterload conditions and therefore can be used to assess RV myocardial function. In the current study, EF-Rt was found to be significantly lower in pectus children than in controls, although EF values were within normal limits and EF-Lft was similar between the two groups. At the same time, IVMA was found to be lower in patients with pectus than in controls, showing that RV contractility is reduced in pectus children. RV functions using quantitative echocardiographic parameters were assessed by Gurkan et al. ${ }^{[31]}$ They reported that the preoperative tricuspid annular plane systolic excursion, $\mathrm{SV}$, and IVMA values were significantly improved in the postoperative period.

We showed that SF-Rt and SF-Lft were lower in pectus children than in controls. A decrease in the SF usually precedes a noticeable decrease in the EF and, similar to EF, is related to ventricular function. Besides RV functions, LV contractility was also affected. E, A, and DT were used for both ventricular diastolic function parameters. Tricuspid valve DT that was found to be prolonged in patients with PE and PAPm was similar in both groups.

\section{Conclusion}

We found that PE can lead to major cardiac problems, not limited only to the RV just below the defect; the LV systolic function also can be affected. We failed to demonstrate a relationship between the severity of $\mathrm{PE}$ and cardiac function. This possibly was due to the limited number of severe cases in our PE group. Clinicians should pay more attention even in mild PE cases due to the markedly affected cardiac functions in these individuals.

Study limitations: In the current study most of the children had a mild and moderate form of the PE. Further studies with larger sample size and a greater number of children with a severe form of PE are warranted to better elucidate the cardiac functions in patients with $P E$.

\section{References}

1. Molik KA, Engum SA, Rescorla FJ, West KW, Scherer LR, Grosfeld JL. Pectus excavatum repair: experience with standard and minimal invasive techniques. J Pediatr Surg 2001; 36: 324-328.

2. Blanco FC, Elliott ST, Sandler AD. Management of congenital chest wall deformities. Semin Plast Surg 2011; 25: 107-116.

3. Williams AM, Crabbe DC. Pectus deformities of the anterior chest wall. Paediatr Respir Rev 2003; 4: 237-242.

4. Fokin AA, Steuerwald NM, Ahrens WA, Allen KE. Anatomical, histologic, and genetic characteristics of congenital chest wall deformities. Semin Thorac Cardiovasc Surg 2009; 21: 44-57.

5. Zhao L, Fenberg MS, Gaides M, Ben-Dov I. Why is exercise capacity reduced in subjects with pectus excavatum? J Pediatr 2000; 136: 163-167.

6. Mocchegiani R, Badano L, Lestuzzi C, Nicolosi GL, Zanuttini D. Relation of right ventricular morphology and function in pectus excavatum to the severity of the chest wall deformity. Am J Cardiol 1995; 76: 941-946.
7. Haller JA Jr, Peters GN, Mazur D, White JJ. Pectus excavatum: a 20-year surgical experience. J Thorac Cardiovasc Surg 1970; 60: 375-383.

8. Jaroszewski DE, Warsame TA, Chandrasekaran K, Chaliki H. Right ventricular compression observed in echocardiography from pectus excavatum deformity. J Cardiovasc Ultrasound 2011; 19: 192-195.

9. Park SY, Park TH, Kim JH, Baek HK, Seo JM, Kim WJ, et al. A case of right ventricular dysfunction caused by pectus excavatum. J Cardiovasc Ultrasound 2010; 18: 62-65.

10. White JA, Fine NM, Shargall Y. Images in cardiovascular medicine. Pectus excavatum with compression of the inferior vena cava: a rare cause of recurrent syncope. Circulation 2009; 120: 1722-1724.

11. Malek MH, Fonkalsrud EW, Cooper CB. Ventilatory and cardiovascular responses to exercise in patients with pectus excavatum. Chest 2003; 124 : $870-882$.

12. Yalamanchili K, Summer W, Valentine V. Pectus excavatum with inspira- 
tory inferior vena cava compression: a new presentation of pulsus paradoxus. Am J Med Sci 2005; 329: 45-47.

13. Haller JA Jr, Loughlin GM. Cardiorespiratory function is significantly improved following corrective surgery for severe pectus excavatum. Proposed treatment guidelines. J Cardiovasc Surg (Torino) 2000; 41: 125-130.

14. Quigley PM, Haller JA Jr, Jelus KL, Loughlin GM, Marcus CLL. Cardiorespiratory function before and after corrective surgery in pectus excavatum. J Pediatr 1996; 128: 638-643.

15. Wynn SR, Driscoll DJ, Ostrom NK, Staats BA, O’Connell EJ, Mottram $\mathrm{CD}$, et al. Exercise cardiorespiratory function in adolescents with pectus excavatum. Observations before and after operation. J Thorac Cardiovasc Surg 1990; 99: 41-47.

16. Mosteller RD. Simplified calculation of body-surface area. N Engl J Med 1987: 22; 1098 .

17. Jaroszewski DE, Fonkalsrud EW. Repair of pectus chest deformities in 320 adult patients: 21 year experce. Ann Thorac Surg 2007; 84: 429-433.

18. Bleeker GB, Steendijk P, Holman ER, Yu CM, Breithardt OA, Kaandorp TA, et al. Assessing right ventricular function: the role of echocardiography and complementary technologies. Heart 2006; 92 Suppl 1: 19-26.

19. Sigalet DL, Montgomery M, Harder J. Cardiopulmonary effects of closed repair of pectus excavatum. J Pediatr Surg 2003; 38: 380-385.

20. Gürsu AH, Karagün BS, Korkmaz O, Gürsu SS, Uçar MA. Correlation between Haller index and echocardiographic and spirometric findings in children with pectus excavatum. Turk Kardiyol Dern Ars 2014; 42: 259-264.

21. Krueger T, Chassot PG, Christodoulou M, Cheng C, Ris HB, Magnusson L. Cardiac function assessed by transesophageal echocardiography during pectus excavatum repair. Ann Thorac Surg 2010; 89: 240-243.

22. Bawazir OA, Montgomery M, Harder J, Sigalet DL. Mid -term evaluation of cardiopulmonary effects of closed repair for pectus excavatum J Pediatr Surg 2005; 40: 863-867.
23. Malek MH, Berger DE, Housh TJ. Cardiovascular function following surgical repair of pectus excavatum; a meta analysis. Chest 2006; 130: 506-516.

24. Lyons HA, Zuhdi MN, Kelly JJ Jr. Pectus excavatum ("funnel breast"), a cause of impaired ventricular distensibility as exhibited by right ventricular pressure pattern. Am Heart J 1955; 50: 921-922.

25. Peterson RJ, Young WG Jr, Godwin JD, Sabiston DC Jr, Jones RH. Noninvasive assessment of exercise cardiac function before and after pectus excavatum repair. J Thorac Cardiovasc Surg 1985; 90: 251-260.

26. Saleh RS, Finn JP, Fenchel M, Moghadam AN, Krishnam M, Abrazado M, et al. Cardiovascular magnetic resonance in patients with pectus excavatum compared with normal controls. J Cardiovasc Magn Reson 2010; 12: 73.

27. Sutherland GR, Stewart MJ, Moran CM, Fleming A, Guell-Peris FJ, et al. Color Doppler myocardial imaging: a new technique for the assessment of myocardial function. J Am Soc Echocardiogr 1994; 7: 441-458.

28. Gorcsan J 3rd, Strum DP, Mandarino WA, Gulati VK, Pinsky MR. Quantitative assessment of alterations in regional left ventricular contractility with color-coded tissue Doppler echocardiography: comparison with sonomicrometry and pressure-volume relations. Circulation 1997; 95: 2423-2433.

29. Urheim S, Edvardsen T, Torp H, Angelsen B, Smiseth OA. Myocardial strain by Doppler echocardiography: validation of a new method to quantify regional myocardial function. Circulation 2000; 102: 1158-1164.

30. Vogel M, Schmidt MR, Kristiansen SB, Cheung M, White PA, Sorensen $\mathrm{K}$, et al. Validation of myocardial acceleration during isovolumic contraction as a novel noninvasive index of right ventricular contractility: comparison with ventricular pressure-volume relations in an animal model. Circulation 2002; 105: 1693-1699.

31. Gürkan U, Aydemir B, Aksoy S, Akgöz H, Tosu AR, Öz D, et al. Echocardiographic assessment of right ventricular function before and after surgery in patients with pectus excavatum and right ventricular compression. Thorac Cardiovasc Surg 2014; 62: 231-235.

Received: 27/04/2016

Accepted: 24/05/2016

Published: 20/06/2016

Disclosure and conflicts of interest:

Conflicts of interest were not reported.

Corresponding author:

Dr. Esra Akyüz Özkan

Mail: uzdresra@gmail.com 